\title{
Development of a Moderate Fidelity Neck-Dissection Simulator
}

\author{
Garrett R. Griffin, MD; Samuel Rosenbaum, BS; Sarah Hecht, BS; Gordon H. Sun, MD
}

Key Words: Surgical simulation, neck dissection.

\section{INTRODUCTION}

Selective neck dissection can be viewed as a "keystone" operation, mastery of which essentially proves the ability to work safely around cranial nerves and the great vessels, access deep neck space infections, and perform smaller "component" procedures like submandibular gland excision. It is a relatively complicated surgery with potentially severe complications including incomplete lymphadenectomy with persistent/ recurrent malignancy, cranial nerve injury, stroke, and high-flow chyle leak. We feel that traditional reading alone is inadequate preparation for a trainee's first several neck dissections as resident surgeon. The tortuous three-dimensional anatomy and process of removing the lymphatic package en bloc are difficult to grasp from text and two-dimensional drawings. A moderate-fidelity neck dissection simulator (NDS) was created to augment book learning.

\section{MATERIALS AND METHODS}

The NDS was built by hand from a mixture of premade and fabricated parts. All components without a place of manufacture mentioned can be purchased at a hardware store. The simulator housing was derived from a Blue Phantom Central

From the Center for Advanced Facial Plastic Surgery, Beverly Hills, California (G.R.G.); University of Michigan School of Medicine, Ann Arbor, Michigan (s.R., S.H.); and Department of Otolaryngology-Head and Neck Surgery, University of Michigan Health System, Ann Arbor, Michigan (G.H.S.), U.S.A.

Editor's Note: This Manuscript was accepted for publication September 14, 2012.

This report was deemed exempt from requiring approval by the institutional review board.

The work was funded by the University of Michigan Clinical Simulation Center.

The authors have no other funding, financial relationships, or conflicts of interest to disclose.

Send correspondence to Garrett R. Griffin, MD, Facial Plastic and Reconstructive Surgery Fellow, The Center for Advanced Facial Plastic Surgery, 9401 Wilshire Boulevard, Beverly Hills, CA 90212.

E-mail: griffin.fpsurgery@gmail.com

DOI: 10.1002/lary.23769
Line Placement Training Model (Blue Phantom, Redmond, WA) with the transparent insert removed (Fig. 1). This created an empty space in the shape of a neck to which additional components could be added. All parts were reversibly added to the Blue Phantom, allowing it to retain its original functionality as a central line trainer. Portions of a Sawbones Full Skull with Fractures (Sawbones, Vashon, WA) were removed with a small circular saw and shaped to form a mock temporal bone comprising the mastoid process, jugular foramen, and hiatus for the internal carotid artery (Fig. 2). This and several other components were secured to the housing using thin (24-28 gauge) wire passed through a 14-gauge Angiocath needle (BD, Franklin Lakes, NJ) that had been passed through the full thickness of the Blue Phantom (Fig. 3). An 18-V Dewalt drill was used to make a hole in the medial, lateral, and inferior walls of the Blue Phantom. A 2-inch long piece of 1.25-inch diameter white

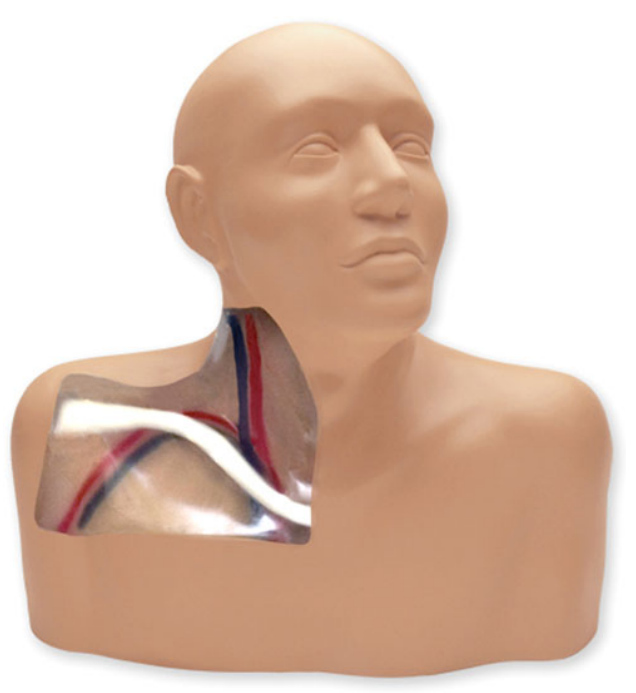

Fig. 1. An image of the Blue Phantom brand Central Line Placement Training Model used as the neck dissection simulator housing. Note that the clear plastic insert must be removed to make room for skeletal, muscular, and neurovascular structures. [Color figure can be viewed in the online issue, which is available at wileyonlinelibrary.com.] 


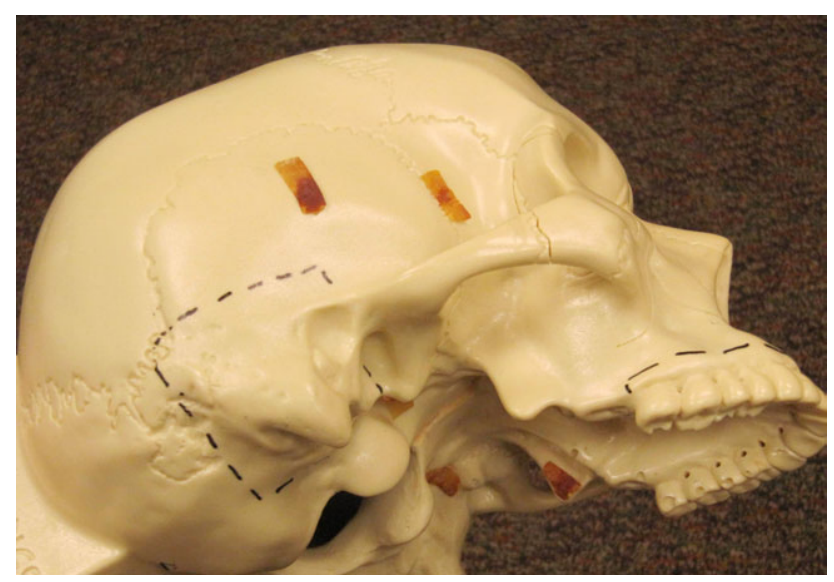

Fig. 2. Sawbones brand Full Skull With Fractures. The mastoid process was removed to form the neck dissection simulator's lateral skull base. The maxillary dental arch was removed to serve as the hyoid bone in the simulator. [Color figure can be viewed in the online issue, which is available at wileyonlinelibrary.com.]

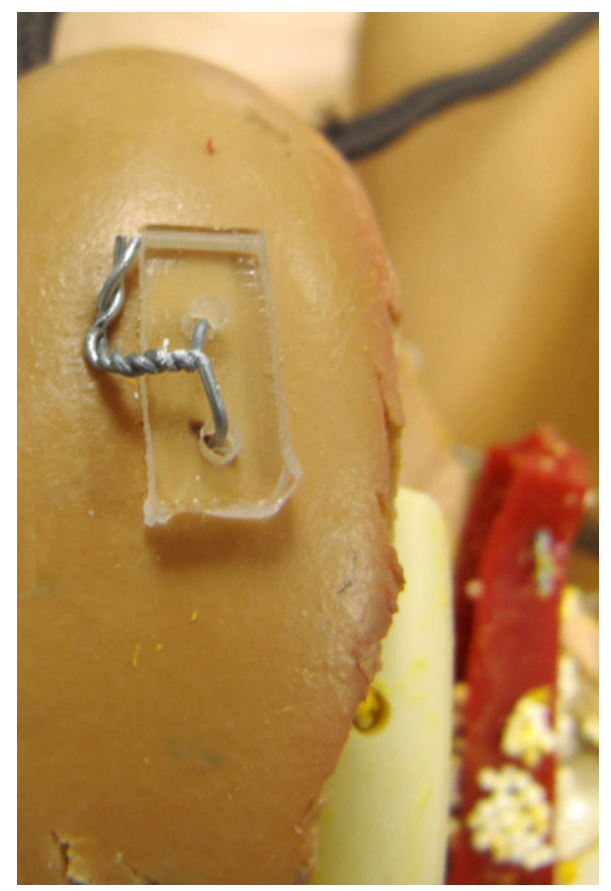

Fig. 3. The skeletal components were able to be secured to the housing using 28-guage wire. A 14-gauge angiocatheter needle was passed through the full thickness of the housing, allowing the wire to be passed through the needle. [Color figure can be viewed in the online issue, which is available at wileyonlinelibrary.com.]

plastic tubing was cut and secured to the hole in the lateral wall with epoxy, thus simulating the acromioclavicular (AC) joint. A piece of 1.0-inch diameter plastic plumbing tubing was then cut such that it could be placed between the "AC joint" and medial wall of the housing under tension, simulating the clavicle. Small brass eye hooks were secured to the "clavicle" medially and laterally to allow attachment of the sternocleido-

Fig. 6. The lateral skull base. Small brass eye hooks were screwed into the skeletal components to serve as attachment points for muscle (Fig. 6). The great vessels, occipital artery, and cranial nerves $\mathrm{XI}$ and XII are also pictured. This image was obtained before we added simulated fat. [Color figure can be viewed in the online issue, which is available at wileyonlinelibrary.com.]

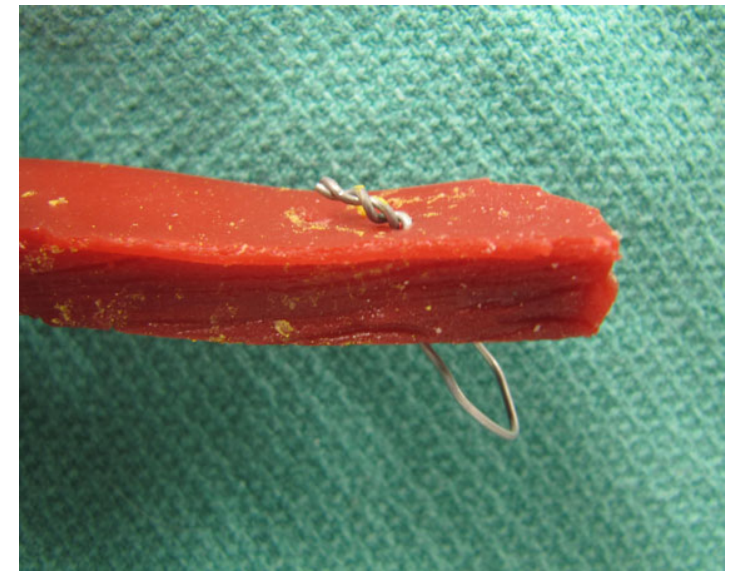

Fig. 4. Pieces of 28-gauge wire were passed through the Sawbones psoas muscle pieces and twisted to create hooks that could be anchored to the brass eye hooks placed into the skeletal components. [Color figure can be viewed in the online issue, which is available at wileyonlinelibrary.com.]

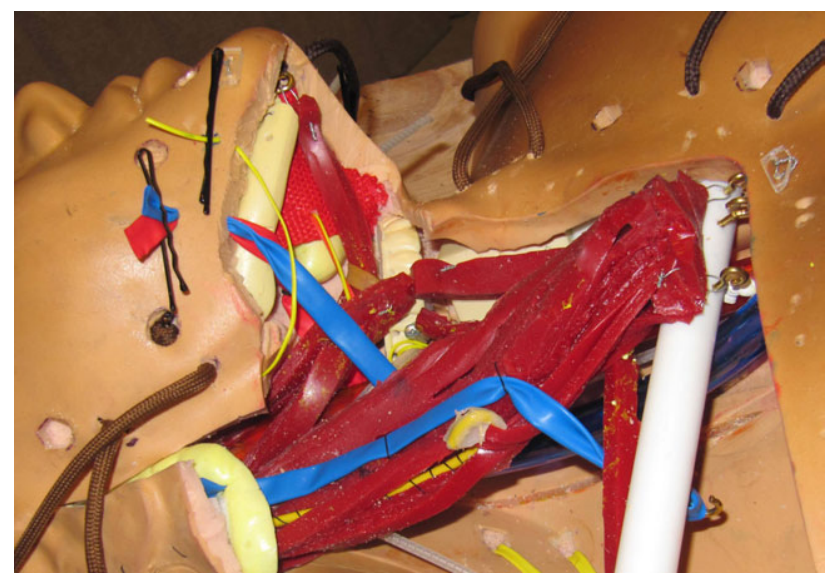

Fig. 5. The fully assembled simulator before adding simulated fat or skin. Vessels that are frequently ligated during selective neck dissection, such as the facial vessels and external jugular vein, are simulated using thin balloons that are an appropriate size and consistency and can be ligated and divided much like real vessels. The sternocleidomastoid, digastric, stylohyoid, mylohyoid, and omohyoid muscles are in place. The marginal and accessory nerves, the cervical rootlets, and the mylohyoid neurovascular bundle are all pictured. The parotid and submandibular glands and clavicle are pictured as well. [Color figure can be viewed in the online issue, which is available at wileyonlinelibrary.com.]

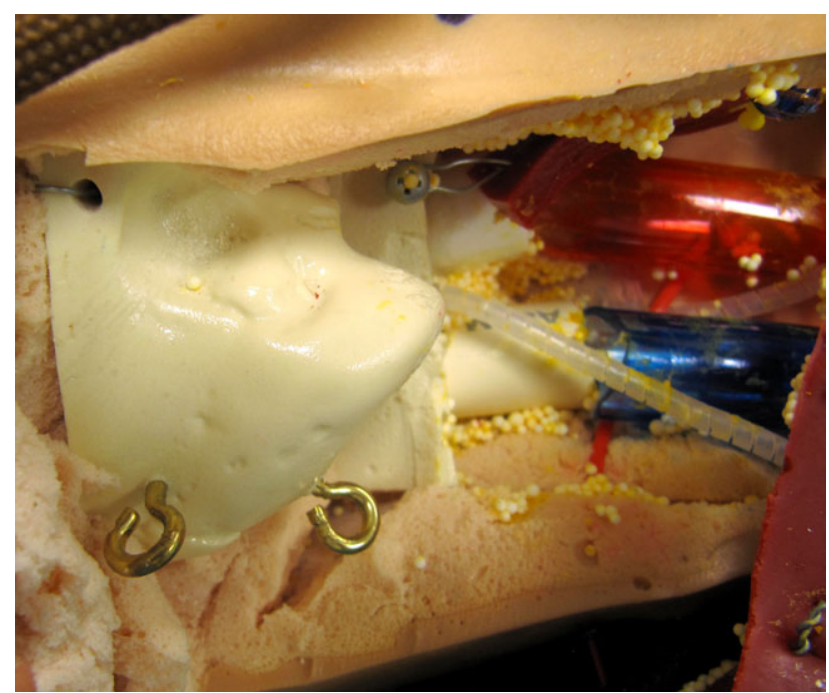




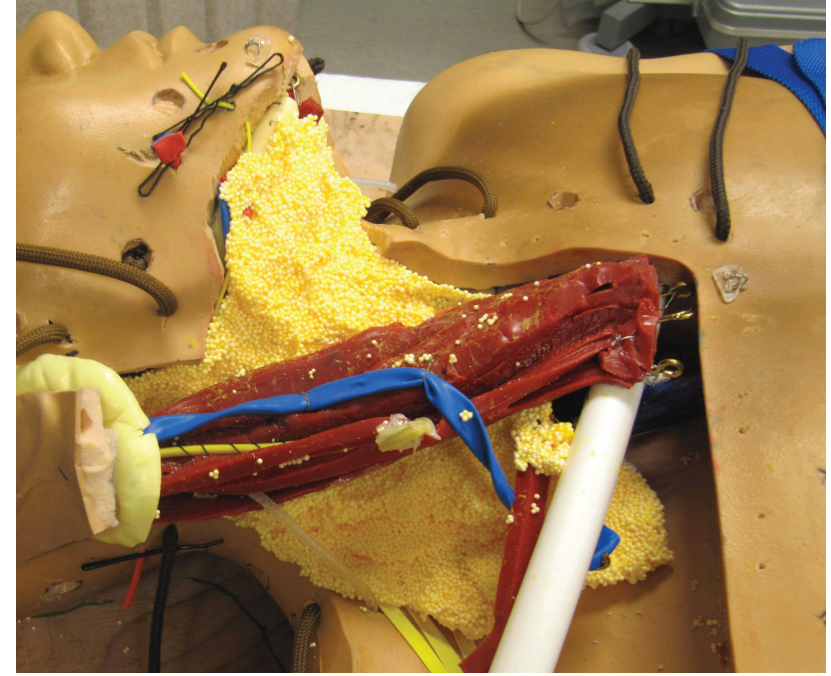

Fig. 7. Once assembly was complete, simulated fat was packed around the deeper neck structures and under the simulated sternocleidomastoid.

mastoid muscle, omohyoid muscle, and external jugular vein, respectively. A trachea, larynx, and hyoid were fashioned from the base and maxillary dental arch of the Sawbones head and secured with 28-gauge wire as described. A utility knife was used to carve a groove from the solid rubber Blue Phantom head, into which a properly sized portion of the Sawbones mandible was placed and secured with wire and tension (Fig. 5 and Fig. 6). This completed the skeletal components of the simulator.

A Psoas Muscle Replacement (Sawbones) was divided with scissors and used to fashion the muscles relevant to neck dissection, including the sternocleidomastoid, digastric, omohyoid, sternohyoid, and stylohyoid muscles. These muscles were secured to screws and eye hooks on the simulated skeleton in anatomically correct locations using small, handmade 28 -gauge wire loops (Fig. 4). The fan-shaped mylohyoid muscle was simu-

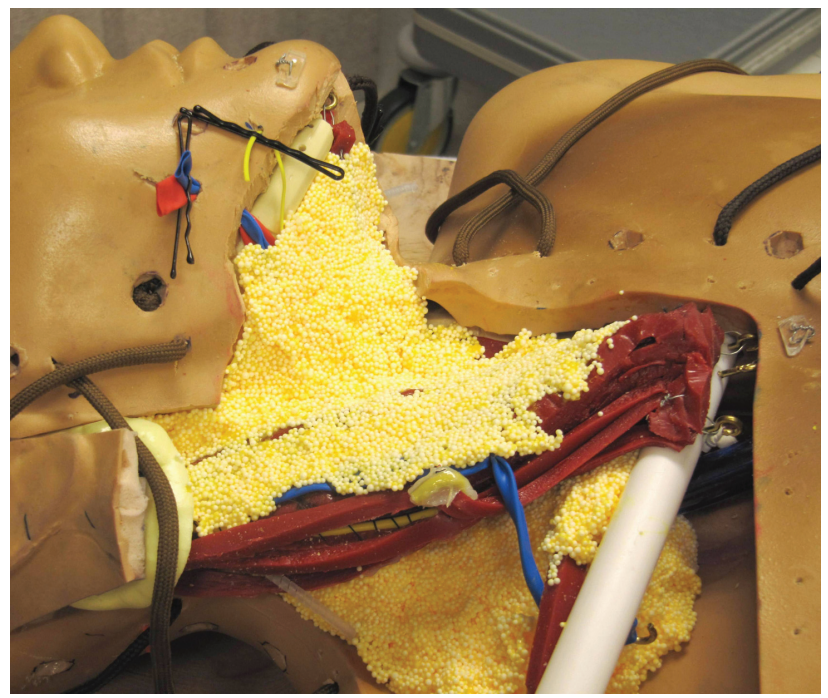

Fig. 8. Simulated fat was then placed thinly over the sternocleidomastoid, external jugular vein, and accessory nerve to minimally disguise them.

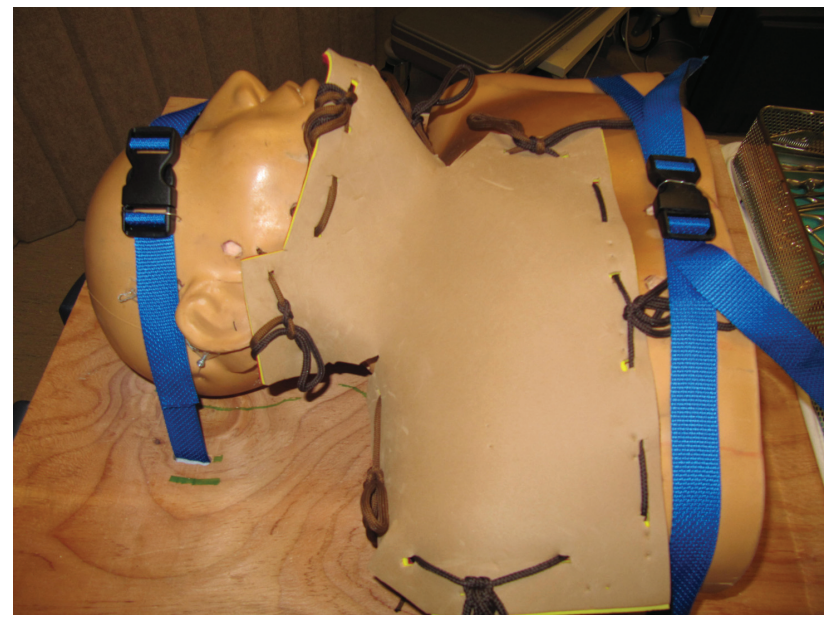

Fig. 9. Completed simulator with skin in place. The simulator was secured to a piece of wood to add weight, helping to resist force exerted during dissection.

lated with a piece of red nylon belt sewn to the mandible and medial wall of the housing with 3-0 silk suture (Fig. 5).

The great vessels were built from 1-inch flexible, translucent aquarium tubing cut to length so that they could be suspended under tension between the Sawbones skull base and the holes in the inferior wall of the housing. Permanent markers added red and blue color. Holes were drilled through the great vessels at the level of the facial artery and vein takeoff, and long "animal" balloons were knotted at one end and passed through the holes in the great vessels to create the facial artery and vein. The external jugular vein was simulated using a balloon as well. The smaller occipital and mylohyoid arteries were simulated with red vessel loops (Fig. 5 and Fig. 6).

The larger cranial nerves (CN XI, CN XII, lingual nerve, vagus nerve, phrenic nerve, and great auricular nerve) were simulated using 12-gauge coiled plastic tubing. This material is the correct diameter and is difficult to locate during dissection, thus creating respect for these critical structures during the simulated neck dissection. The smaller marginal mandibular, ansa cervicalis, and mylohyoid nerves were simulated with yellow vessel loops. Yellow balloons emerging from the posterior

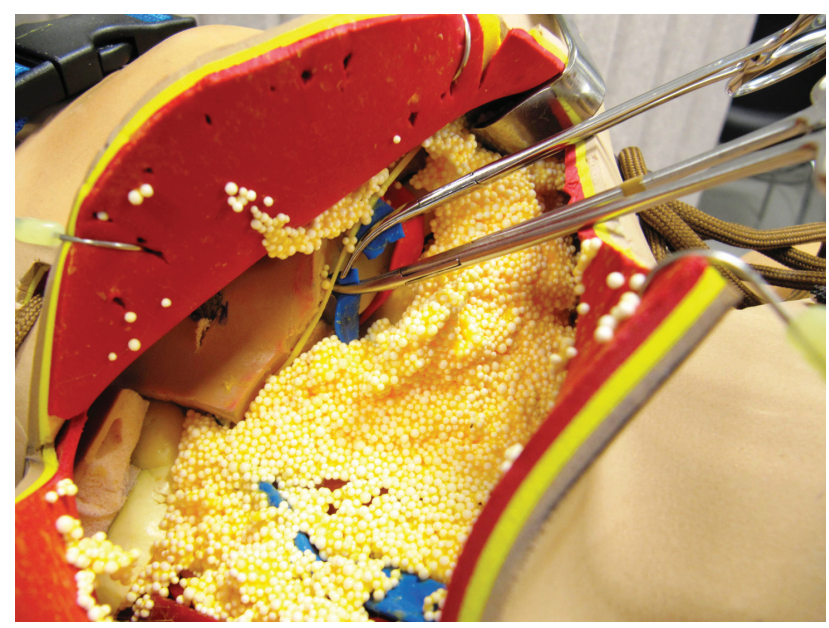

Fig. 10. A selective neck dissection could be performed with real surgical instruments. Subplatysmal flaps have been elevated and retracted. The marginal nerve has been dissected out, and the facial vein is in the process of being ligated. 
wall of the Blue Phantom housing were used to simulate the cervical rootlets. The phrenic nerve was placed in a slit in the posterior wall of the Blue Phantom housing to emulate its location below the deep layer of the deep cervical fascia. Simulated nerves and blood vessels were held in place by passing them through small holes drilled in the Blue Phantom, with epoxy, or under tension. Bobby pins are an inexpensive way to "lock" these structures in place (Fig. 5).

Yellow molding compound (Floam; NSI International, New York, NY) is used to simulate fat and lymphoareolar tissue. It is placed around the skeletal, muscular, and neurovascular components once they are in place. This material obscures the anatomy and can be dissected much like the fat in a living human (Fig. 7 and Fig. 8).

The skin was built from thin beige, yellow, and red layers of foam (Creatology Fun Foam, purchased at Michaels store) glued together with Loctite spray adhesive. A scalpel was used to cut small holes in the foam in appropriate locations. Shoelaces were secured to the Blue Phantom housing and used to tie the skin down to the simulator (Fig. 9).

Four first-year and four second-year otolaryngology residents performed a selective neck dissection of levels I to IV on the simulator with the first author, a research fellow with no impact on resident performance evaluations. Real surgical instruments were used during the dissection. For example, a scalpel was used for the skin incision, and nerves were dissected as necessary using tenotomy scissors and forceps (Fig. 10). Muscles were retracted with appropriately sized retractors. Large vessels were ligated and divided using hemostats and suture. Cautery was not used for obvious reasons; instead, the blunt side of a no. 9 dental elevator was used to dissect through the simulated fat.

\section{DISCUSSION}

Modern medical practice has introduced several new obstacles to the traditional apprenticeship model of surgical education. Work hour restrictions and heightened focus on surgical efficiency and prevention of errors have made the training of surgeons more challenging than ever.

Simulation is one method by which surgical residency programs have sought to supplement training received in the operating room. Most surgical simulators that have been described involve endoscopic procedures like bronchoscopy or laparoscopy. ${ }^{1-3}$ It is much more challenging to simulate soft-tissue procedures like neck dissection. A thorough literature search revealed only one complex soft-tissue surgical simulator. ${ }^{4}$ There has been some early development of computer-based soft-tissue simulators with haptic feedback, but this technology requires further development. ${ }^{5}$ Cadaver and live-animal dissections provide the highest-fidelity soft-tissue simulations but can be expensive, space intensive, and sociopolitically delicate.

In this manuscript, the authors describe a low-cost moderate-fidelity neck dissection simulator. Although the Blue Phantom housing currently costs nearly $\$ 3,000$, it can retain its original purpose as a central line simulator and is likely already owned by many academic simulation centers. The other components cost less than $\$ 250$ in total. Our simulator was purposely built in such a way that the anatomic pieces can be removed and replaced. This allows components like blood vessels to be realistically manipulated and divided during simulation and then "refreshed" for the next iteration. Perhaps even more importantly, it allows trainees to build the simulator prior to using it. For example, we think that the act of threading the hypoglossal nerve between the internal jugular vein and carotid artery likely improves retention of this important anatomic relationship. This idea deserves further study.

It is difficult to validate a surgical simulator because of the relatively low number of otolaryngology trainees at a single institution. We hope that this manuscript will allow other centers to build similar simulators, which in turn would allow a multi-institutional validation study. Ideally, selective neck dissection on a live surgical patient would be used as a validation measure. This in turn will likely require the development of an Objective Structured Assessment of Technical Skill for neck dissection, which does not currently exist.

\section{CONCLUSION}

This is the first published neck dissection simulator and one of the first complex soft-tissue simulators described in the medical literature. In the future, it would be ideal to have multi-institutional studies that focus on maximizing the simulator's realism and on evaluating its validity and effect on operating room performance.

\section{Acknowledgment}

The authors thank the University of Michigan Clinical Simulation Center for funding this project.

\section{BIBLIOGRAPHY}

1. Griffin GR, Hoesli B, Thorne MC. Validity and efficacy of a pediatric airway foreign body training course in resident education. Ann Oto Rhinol Laryngol 2011;120:635-640.

2. Bittner JG, Marks JM, Dunkin BJ, Richards WO, Onders RP, Mellinger JD. Resident training in flexible gastrointestinal endoscopy: a review of current issues and options. J Surg Ed 2007;64:399-409.

3. Seymour NE, Gallagher AG, Roman SA, et al. Virtual reality training improves operating room performance: results of a randomized, doubleblinded study. Ann Surg 2002;236:458-464.

4. Stern C, Oliker A, Napier Z, et al. Integration of surgical simulation in plastic surgery residency training. Stud Health Technol Inform 2012; 173:497-499.

5. Schendel S, Montgomery K, Sorokin A, Lionetti G. A surgical simulator for planning and performing repair of cleft lips. J Craniomaxillofac Surg $2005 ; 33: 223-228$. 